\title{
Pancreatic Schwannoma Diagnosed by Endoscopic Ultrasound-guided Fine-needle Aspiration
}

\author{
Taro Hanaoka ${ }^{1}$, Kosuke Okuwaki ${ }^{1}$, Hiroshi Imaizumi ${ }^{1,2}$, Yusuke Imawari ${ }^{1,2}$, Tomohisa Iwai ${ }^{1}$, \\ Hiroshi Yamauchi ${ }^{1}$, Rikiya Hasegawa ${ }^{1}$, Kai Adachi ${ }^{1}$, Masayoshi Tadehara ${ }^{1}$, Takahiro Kurosu ${ }^{1}$, \\ Masafumi Watanabe ${ }^{1}$, Akihiro Tamaki ${ }^{1}$, Mitsuhiro Kida ${ }^{1}$ and Wasaburo Koizumi ${ }^{1}$
}

\begin{abstract}
:
A schwannoma is a tumor originating from Schwann cells. It is occasionally observed in the abdominal viscera in the form of a submucosal tumor derived from the gastric or duodenal muscularis propria. To date, only a few studies have reported on pancreatic schwannomas. Furthermore, very few patients are preoperatively diagnosed with pancreatic schwannoma because of the lack of established imaging characteristics distinguishing this type of schwannoma from other conditions. We herein report the first English publication of pancreatic schwannoma in which surgery was avoided because a pathological diagnosis was made solely on the basis of endoscopic ultrasound-guided fine-needle aspiration findings.
\end{abstract}

Key words: pancreatic schwannoma, pancreatic neoplasm, endoscopic ultrasound-guided fine-needle aspiration

(Intern Med 60: 1389-1395, 2021)

(DOI: 10.2169/internalmedicine.6129-20)

\section{Introduction}

A schwannoma is a tumor originating from Schwann cells and was first reported by Verocay et al. in 1910 (1). Although a schwannoma can develop in any part of the human body, frequent sites of tumor growth include the extremities, body trunk, head and neck, and retroperitoneum (2). It is occasionally observed in the abdominal viscera in the form of a submucosal tumor, which is derived from the gastric or duodenal muscularis propria. To date, only a few studies have reported on pancreatic schwannomas. Furthermore, an extremely small number of patients are preoperatively diagnosed with pancreatic schwannoma because of the lack of established imaging characteristics that help distinguish this type of schwannoma from other conditions.

We herein report a case of pancreatic schwannoma in which surgery was avoided because a pathological diagnosis was made solely on the basis of endoscopic ultrasound (EUS)-guided fine-needle aspiration (FNA) findings. An imaging-based diagnosis was challenging, as nonspecific imaging findings were observed.

\section{Case Report}

A 73-year-old woman was receiving treatment for hypertension and hyperlipidemia. She had been found to have an enlarged pancreatic duct on abdominal ultrasonography, which was performed during a health examination. An 8$\mathrm{mm}$ low-echogenic mass with poor blood flow was observed in the pancreatic body on EUS performed at an outside hospital (Fig. 1A). The caudal pancreatic duct diameter was 2 $\mathrm{mm}$. On contrast-enhanced EUS, the pancreatic mass showed an avascular pattern in the initial phase. As typical findings of ductal adenocarcinoma (such as dilation of the distal pancreatic duct) were not observed, the patient did not undergo any intervention and was only placed under observation.

Two months thereafter, EUS was repeated. An anechoic area with suspected cystic changes, which had not been ob-

${ }^{1}$ Department of Gastroenterology, Kitasato University School of Medicine, Japan and ${ }^{2}$ Department of Gastroenterology, JCHO Sagamino Hospital, Japan

Received: August 17, 2020; Accepted: September 17, 2020; Advance Publication by J-STAGE: November 30, 2020

Correspondence to Dr. Kosuke Okuwaki, kokuwaki@kitasato-u.ac.jp 

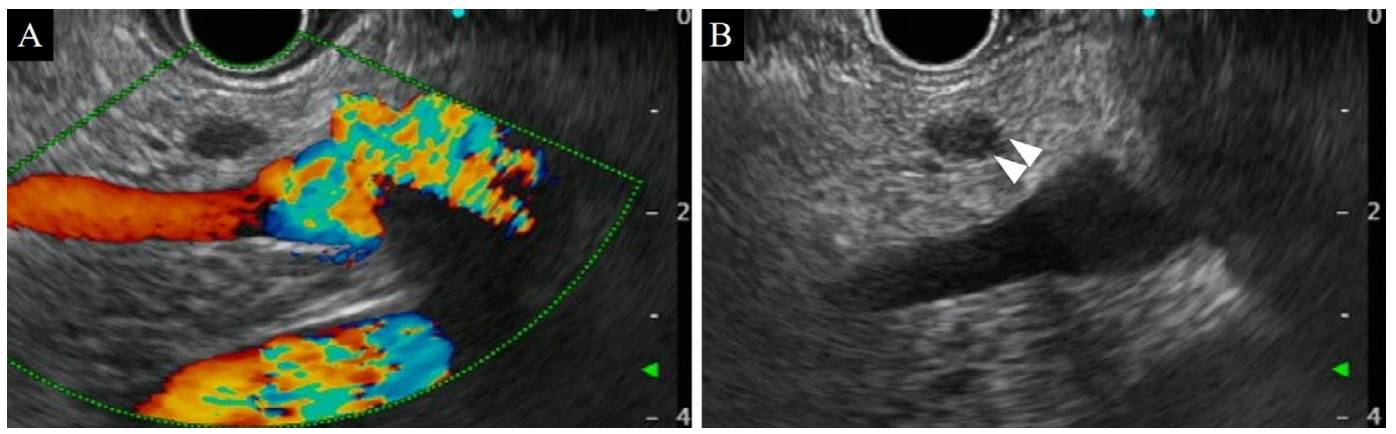

Figure 1. Endoscopic ultrasound (EUS). A: An 8-mm low-echogenic mass with poor blood flow is observed in the pancreatic body on initial EUS. B: EUS repeated two months later reveals an anechoic area (arrowhead) suspected of being a cystic change on the boundary of the tail side of the mass, which had not been observed previously.

Table 1. Clinical Characteristics.

\begin{tabular}{lc}
\hline White blood cells $(/ \mu \mathrm{L})$ & $3.7 \times 10^{3}$ \\
Red blood cells $(/ \mu \mathrm{L})$ & $4.37 \times 10^{6}$ \\
Hemoglobin $(\mathrm{g} / \mathrm{dL})$ & 14.1 \\
Platelet $(/ \mu \mathrm{L})$ & $17.4 \times 10^{4}$ \\
Total bilirubin (mg/dL) & 1.0 \\
AST (IU/L) & 21 \\
ALT (IU/L) & 21 \\
LDH (IU/L) & 170 \\
ALP (IU/L) & 190 \\
$\gamma$-GTP (IU/L) & 17 \\
Total protein (g/dL) & 6.6 \\
Albumin (g/dL) & 4.2 \\
Urea nitrogen (mg/dL) & 12.2 \\
Creatinine (mg/dL) & 0.64 \\
Sodium (mEq/L) & 141 \\
Potassium (mEq/L) & 4.0 \\
CEA (ng/mL) & 1.4 \\
CA19-9 (IU/mL) & 12 \\
DUPAN-2 (IU/mL) & $<25$ \\
Span-1 (IU/mL) & 9.9 \\
Elastase-1 (ng/dL) & $<80$ \\
NSE (ng/mL) & 17.9 \\
proGRP (pg/mL) & 42.2 \\
\hline AST: aspartate aminotransferase, ALT: ala- \\
nine aminotransferase, LDH: lactate dehy- \\
drogenase, ALP: alkaline & phosphatase, \\
$\gamma$-GTP: $\gamma$-glutamyl & transpeptidase, CEA: \\
carcinoembryonic antigen, CA19-9: carbo- \\
hydrate antigen 19-9, DUPAN-2: duke pan- \\
creatic monoclonal antigen type 2, Span-1: \\
s-pancreas antigen-1, NSE: neuron-specific \\
enolase, proGRP: pro-gastrin-releasing pep- \\
tide \\
\end{tabular}

served previously, was found in the boundary of the pancreatic tail (Fig. 1B). A high signal intensity was observed on diffusion-weighted magnetic resonance imaging (MRI). As the possibility of small pancreatic cancer could not be ruled out, the patient was referred to our hospital for a further examination.

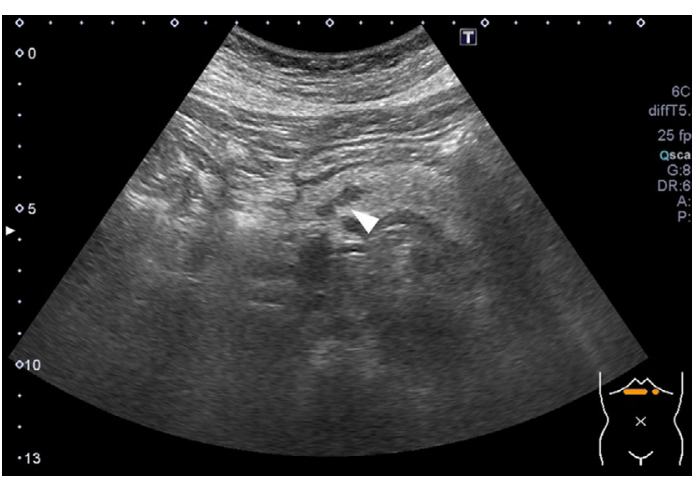

Figure 2. Abdominal ultrasound. A bilocular low-echogenic mass (arrowhead) with clear margins and a major axis of $\mathbf{1 0}$ $\mathrm{mm}$ is observed in the pancreatic body.

There were no abnormalities in the patient's vital signs or on physical examination. The patient's laboratory test results are shown in Table 1. Neuron-specific enolase $(17.9 \mathrm{ng} / \mathrm{mL})$ was slightly elevated. A bilocular low-echogenic mass with clear margins and a major axis of $10 \mathrm{~mm}$ was observed in the pancreatic body on abdominal ultrasound (Fig. 2). A 9$\mathrm{mm}$, low-density area with relatively clear margins was observed in the arterial phase of dynamic computed tomography (CT). Compared to the surrounding pancreatic parenchyma, the area was contrasted strongly in the equilibrium phase (Fig. 3). The pancreatic mass was hypo-intense on T1-weighted MRI, slightly hyper-intense on T2-weighted MRI, and hyper-intense on diffusion-weighted MRI. The cystic component was not clear on MRI. No abnormalities were observed in the formation of the major pancreatic duct on magnetic resonance cholangiopancreatography (MRCP) (Fig. 4). Based on these imaging findings, pancreatic cancer without involvement of the main pancreatic duct, pancreatic neuroendocrine neoplasm, and solid-pseudopapillary neoplasm (SPN) were considered as potential diagnoses.

To make a definitive diagnosis pathologically, we performed an EUS-FNA. A 22-gauge biopsy needle (Acquire $^{\mathrm{TM}}$; Boston Scientific, Marlborough, USA) was used, and a puncture was performed twice via a trans-gastric ac- 


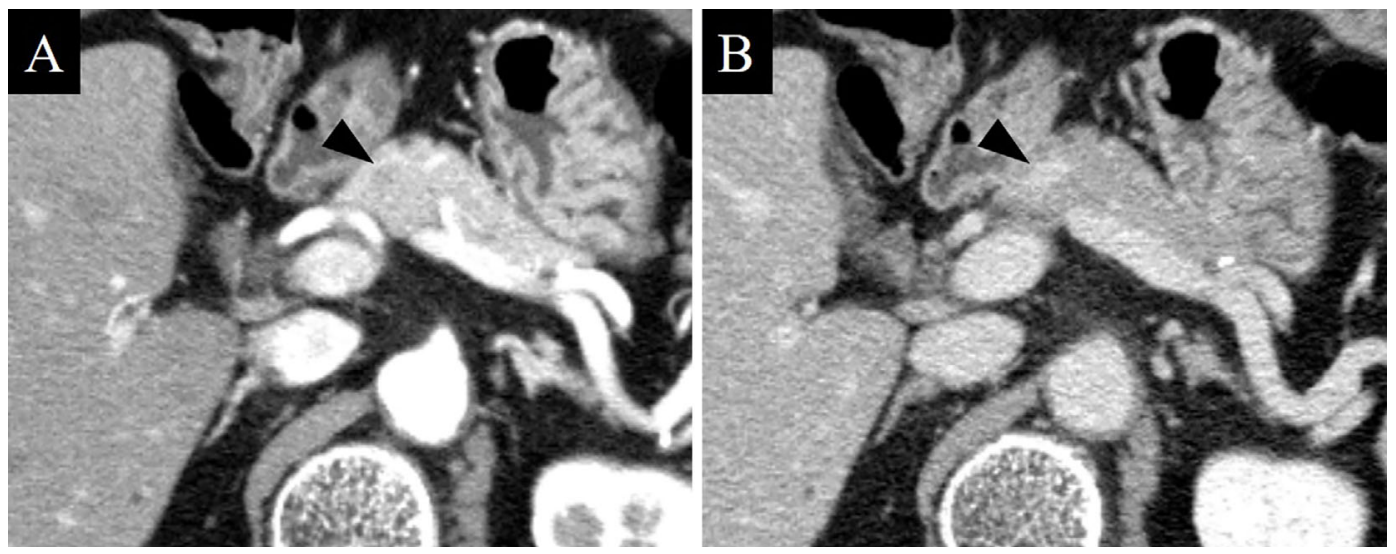

Figure 3. Dynamic computed tomography. A: A 10-mm low-density area (black arrowhead) with relatively clear margins is observed in the pancreatic body in the arterial phase of dynamic computed tomography. B: Compared with the surrounding pancreatic parenchyma, the mass (black arrowhead) shows strong contrast in the equilibrium phase.
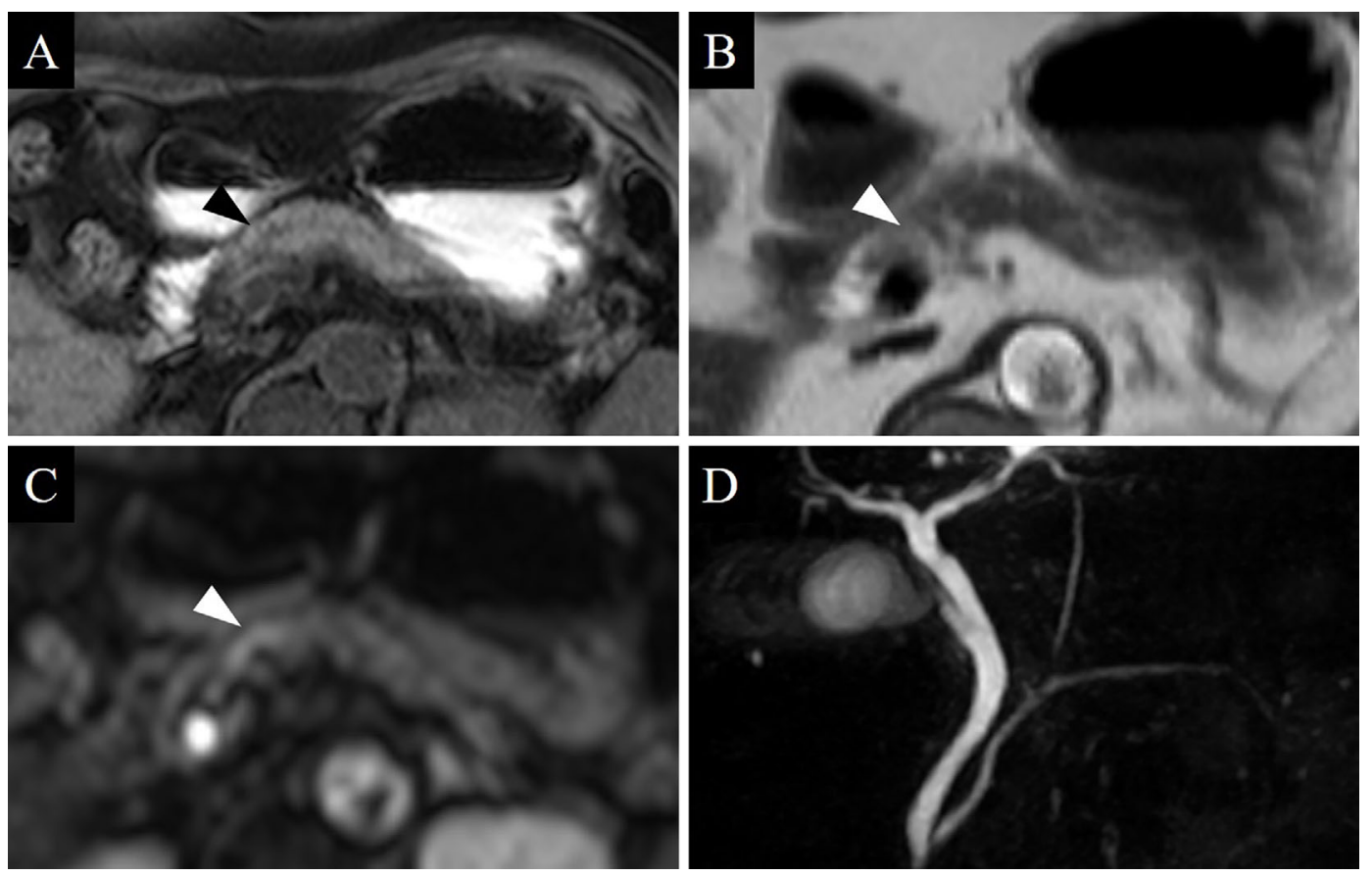

Figure 4. Magnetic resonance imaging and magnetic resonance cholangiopancreatography (MRCP). A: A T1-weighted image shows a low signal intensity (arrowhead) in the pancreatic body. B: A T2-weighted image shows a slightly high signal intensity (arrowhead) in the pancreatic body. C: A diffusion-weighted image shows a high signal intensity (arrowhead) in the pancreatic body. D: An MRCP image shows no abnormalities in the formation of the major pancreatic duct.

cess route under 20-cc suction pressure (Fig. 5). Pathological specimens were acquired via sample isolation by stereomicroscopy, as previously reported (3). Histopathological findings indicated palisading proliferation of spindle cells and nuclear palisading. The neoplastic cells were positive for S-100 protein and neural cell adhesion molecule and negative for $\alpha$-smooth muscle actin, heavy caldesmon, and CA19-9. The cells had an MIB-1 labeling index of less than $1 \%$. Based on these findings, we pathologically diagnosed the patient with pancreatic schwannoma, a benign tumor (Fig. 6).
The patient was observed with no surgical requirements. No significant changes were noted during the six-month observation period following the definitive diagnosis.

\section{Discussion}

Schwannomas can be divided into two histopathological types: highly cellular Antoni type A and sparsely cellular Antoni type B. Schwannoma cells are strongly positive for S-100 proteins, vimentins, and CD56, and negative for cytokeratins, AE1/AE3, desmin, smooth muscle myosins, 


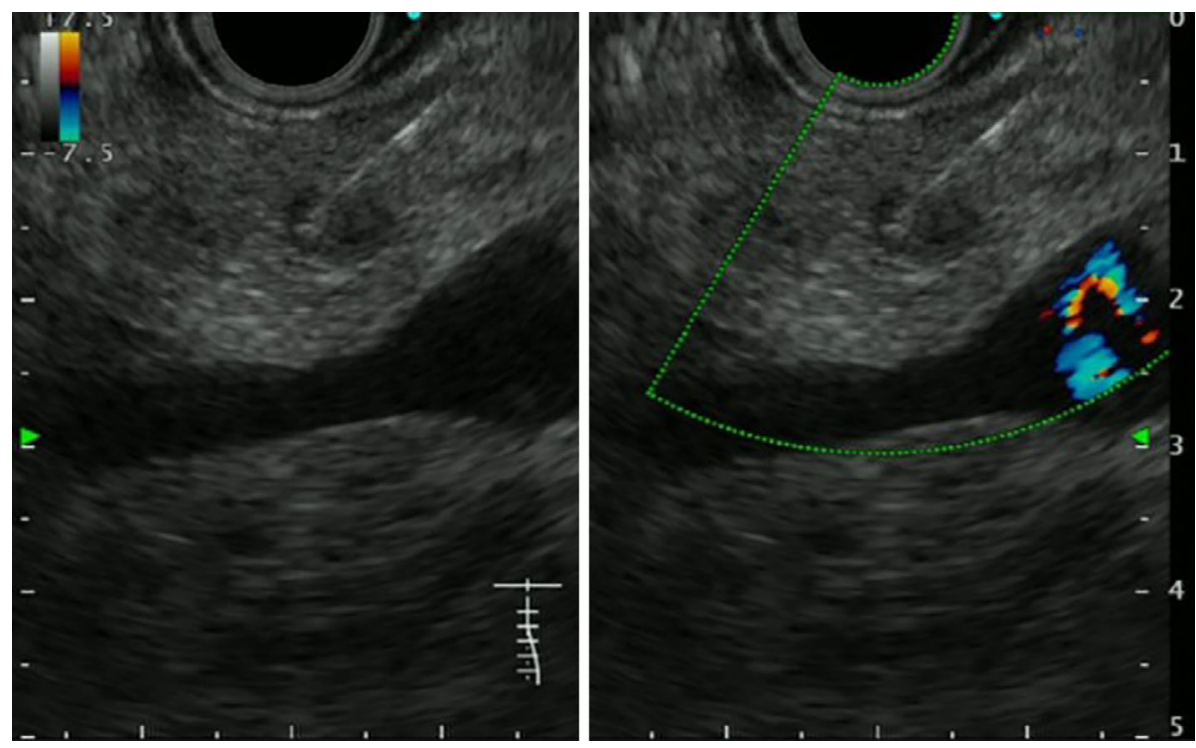

Figure 5. Endoscopic ultrasound-guided fine-needle aspiration. A 22-gauge needle (Acquire ${ }^{\mathrm{TM}}$; Boston Scientific) is used via a trans-gastric access route.


Figure 6. Hematoxylin and Eosin $(\mathrm{H} \& \mathrm{E})$ staining and immunostaining. All pathological specimens were acquired via sample isolation by stereomicroscopy. A: H\&E staining $\times 100$. Antoni type $B$ areas (circle) are sparsely cellular with few vessels. B: H\&E staining $\times 200$. Palisading proliferation of spindle cells and nuclear palisading is observed. C: Immunostaining (S-100 protein) $\times 100$ shows positive results. D: An MIB-1 labeling index of less than $1 \%$ is observed.

\section{CD34, and CD117 (4).}

Imaging can be performed to diagnose a pancreatic schwannoma, including ultrasonography, CT, and MRI, which are also used to diagnose other pancreatic tumors. A mixture of solid components and cystic components is fre- quently observed on CT (5), reflecting the histological characteristics of schwannomas. As Antoni type A areas are highly cellular, they appear as solid components. On contrast CT, these areas are uneven due to the development of small-vessel components. Antoni type B areas, which are 
Table 2. Reports of Pancreatic Schwannomas with a Size of up to $20 \mathrm{~mm}$.

\begin{tabular}{|c|c|c|c|c|c|c|c|c|c|}
\hline Case & Reference & $\begin{array}{l}\text { Age/ } \\
\text { sex }\end{array}$ & Symptoms & Location & $\begin{array}{c}\text { Size } \\
(\mathbf{m m})\end{array}$ & $\begin{array}{l}\text { Primary } \\
\text { diagnosis }\end{array}$ & EUS-FNA & Treatment & $\begin{array}{c}\text { Follow-up } \\
\text { (months) }\end{array}$ \\
\hline 1 & 8 & $71 / \mathrm{M}$ & $\begin{array}{l}\text { Abdominal } \\
\text { pain }\end{array}$ & Head & 15 & Cystic neoplasm & N/A & Enucleation & 10 \\
\hline 2 & 9 & $72 / \mathrm{M}$ & $\begin{array}{l}\text { Abdominal } \\
\text { pain }\end{array}$ & $\begin{array}{c}\text { Head and } \\
\text { body }\end{array}$ & 10 & Pancreatic mass & N/A & Surgery & N/A \\
\hline 3 & 10 & $37 / M$ & Asymptomatic & Body & 16 & Pancreatic mass & Successful & Surgery & N/A \\
\hline 4 & 11 & $54 / \mathrm{F}$ & $\begin{array}{l}\text { Abdominal } \\
\text { pain }\end{array}$ & Head & 14 & Pancreatic mass & N/A & PD & N/A \\
\hline 5 & 12 & $63 / \mathrm{F}$ & $\begin{array}{l}\text { Abdominal } \\
\text { pain }\end{array}$ & Tail & 10 & NR & N/A & DP & N/A \\
\hline 6 & 13 & $55 / \mathrm{F}$ & Asymptomatic & Neck & 10 & Pancreatic mass & Successful & Follow-up & 3 \\
\hline 7 & 14 & $83 / \mathrm{M}$ & Asymptomatic & Body & 20 & Pancreatic mass & Successful & Follow-up & N/A \\
\hline 8 & 15 & $59 / \mathrm{F}$ & Asymptomatic & Body & 16 & $\begin{array}{c}\text { Pancreatic } \\
\text { cystadenoma }\end{array}$ & Unsuccessful & $\mathrm{CP}$ & 53 \\
\hline 9 & 16 & $53 / \mathrm{M}$ & Asymptomatic & Body & 18 & Pancreatic mass & Successful & Follow-up & N/A \\
\hline 10 & 17 & $59 / \mathrm{F}$ & $\begin{array}{l}\text { Abdominal } \\
\text { distention }\end{array}$ & Head & 20 & SPN, NEN, SCN & N/A & SSPPD & 10 \\
\hline 11 & 18 & $78 / \mathrm{F}$ & Asymptomatic & Body & 17 & Pancreatic mass & Successful & Follow-up & 11 \\
\hline 12 & 19 & $54 / \mathrm{F}$ & Asymptomatic & $\begin{array}{c}\text { Head and } \\
\text { body }\end{array}$ & 20 & SPN & Unsuccessful & DP & N/A \\
\hline 13 & 20 & $37 / M$ & $\begin{array}{l}\text { Abdominal } \\
\text { pain }\end{array}$ & Body & 13 & NR & Successful & $\mathrm{CP}$ & 5 \\
\hline 14 & 20 & $43 / \mathrm{F}$ & Asymptomatic & Uncinate & 16 & IPMN & Unsuccessful & PD & 14 \\
\hline 15 & 21 & $44 / \mathrm{F}$ & Asymptomatic & Uncinate & 13 & Pancreatic mass & Successful & Follow-up & 48 \\
\hline 16 & 22 & $55 / \mathrm{F}$ & $\begin{array}{l}\text { Abdominal } \\
\text { distention }\end{array}$ & Body & 20 & $\begin{array}{c}\text { Pancreatic } \\
\text { cystadenoma }\end{array}$ & N/A & DP & 12 \\
\hline 17 & 23 & 79/M & Asymptomatic & Body & 9 & NEN, SPN & Successful & Follow-up & 36 \\
\hline 18 & Our case & $73 / \mathrm{F}$ & Asymptomatic & Body & 10 & $\begin{array}{c}\text { NEN, SPN, } \\
\text { pancreatic cancer }\end{array}$ & Successful & Follow-up & 3 \\
\hline
\end{tabular}

N/A: not available, NR: not reported, SPN: solid pseudopapillary neoplasm, NEN: neuroendocrine neoplasm, SCN: serous cystic neoplasm, IPMN: intraductal papillary mucinous neoplasm, PD: pancreatoduodenectomy, SSPPD: subtotal stomach-preserving pancreatoduodenectomy, DP: distal pancreatectomy, CP: central pancreatectomy

sparsely cellular with few vessels, appear as poorly contrasted pseudo-cysts. Furthermore, a previous study found that intravascular thrombosis tends to occur in Antoni B areas, leading to necrosis and thereby resulting in the formation of cysts (6). An area with a mixture of such solid components and cystic components is also observed on ultrasonography (5). A low signal intensity on T1-weighted images and a high signal intensity on T2-weighted images are often observed on MRI, whereas tumors are usually contrasted gradually on T1-weighted images of gadolinium ethoxybenzyl diethylenetriamine pentaacetic acid contrastenhanced MRI (7). However, these imaging findings can also be observed in other pancreatic tumors and are not diagnostic for pancreatic schwannomas. In particular, findings showing a mixture of solid components and cystic components in a mass with smooth margins can also be seen in SPNs, pancreatic neuroendocrine tumors with cystic changes, and pancreatic epithelial cysts. Therefore, it is difficult to make a diagnosis of pancreatic schwannoma solely based on imaging findings.

In the present case, an anechoic area suspected of being a cystic change was observed to have formed during a short observation period. Furthermore, varying imaging findings were observed on EUS at our hospital; for example, there was a morphological change from unilocular to bilocular type. This may have been because the previously mentioned Antoni A and B areas were both present in a complex manner within the mass. Therefore, consistent with previous studies, we also had difficulty making an imaging-based diagnosis. However, we managed to make a pathological diagnosis of pancreatic schwannoma based on the EUS-FNA findings.

Despite the small size of the tumor in the present case, EUS-FNA allowed for a pathological diagnosis. We searched PubMed for cases of schwannomas of up to $20 \mathrm{~mm}$ and found only 17 cases [Table 2 (8-23)]. The mean age of the 18 patients, including the present case, was 59.4 years old. Of these patients, 7 were men, and 11 were women. Seven patients exhibited symptoms. In particular, the tumor was in the pancreatic head in three patients, pancreatic head and body in two patients, pancreatic neck in one patient, pancreatic body in nine patients, pancreatic tail in one patient, and uncinate process in two patients. None of these patients were diagnosed with schwannoma based on imaging findings. The differential diagnoses were varied and included serous cystic neoplasm, SPN, neuroendocrine tumor, and in- 
traductal papillary mucinous neoplasm. Among these 18 patients, 12 underwent EUS-FNA, of whom 9 were diagnosed with schwannoma. Observation was conducted in 7 patients, and surgery was performed in 11 patients. Even when patients had small tumors, diagnostic surgery was performed for the majority, which differs from our management. Observation was conducted in seven of nine patients who were pathologically diagnosed through EUS-FNA, including our patient. All seven cases of pancreatic schwannoma diagnosed by EUS-FNA without surgical resection showed positive staining for S-100 protein. EUS-FNA was performed using a 25-gauge needle in four patients and a 22-gauge needle in three patients. A biopsy needle was used in three patients.

According to Bruno et al. (21), the diagnostic rate of pancreatic schwannoma through EUS-FNA is $52.9 \%$, which is lower than that of other pancreatic tumors. However, studies have found that using the recently developed Franseen needle enables the collection of more tissue than existing puncture needles $(24,25)$. Changing the needle type may help improve the diagnostic rate of small tumors, such as that in the present case. EUS-FNA is useful for making a pathological diagnosis of small pancreatic tumors, which can facilitate the observation and selection of minimally invasive surgery. Therefore, we recommend EUS-FNA for pancreatic tumors with nonspecific imaging findings that may be pancreatic schwannomas, such as the present case.

As the present patient had a small, asymptomatic tumor with a low MIB-1 labeling index, the lesion was followed up without surgical resection. If the tumor grows, it may compress the pancreatic duct, and if it develops into a large tumor, it may be found to be a malignant schwannoma (26). Therefore, careful monitoring is required.

In summary, we herein report the first English publication of pancreatic schwannoma diagnosed using EUS-FNA with a Franseen needle and immunohistochemistry without surgical resection. A definitive diagnosis is often made after surgery is performed. However, it is possible to avoid major surgery if the pathological diagnosis is made preoperatively. In recent years, the accuracy of EUS-FNA-based diagnoses of pancreatic tumors has improved. Consequently, the proactive use of EUS-FNA for nonspecific pancreatic tumors is recommended.

\section{The authors state that they have no Conflict of Interest (COI).}

\section{References}

1. Verocay J. Zur kenntnis der neurofibrome. Beitr Pathol Anat Allg Pathol 48: 1-69, 1910 (in Czech).

2. Akiyoshi T, Ueda Y, Yanai K, et al. Melanotic schwannoma of the pancreas: report of a case. Surg Today 34: 550-553, 2004.

3. Masutani H, Okuwaki K, Kida M, et al. On-site stereomicroscope quality evaluations to estimate white core cutoff lengths using EUS-FNA biopsy sampling with 22-gauge needles. Gastrointest Endosc 90: 947-956, 2019.

4. Weiss SW, Langloss JM, Enzinger FM. Value of S-100 protein in the diagnosis of soft tissue tumors with particular reference to benign and malignant Schwann cell tumors. Lab Invest 49: 299-308, 1983.

5. Suzuki S, Kaji S, Koike N, et al. Pancreatic schwannoma: a case report and literature review with special reference to image features. JOP 11: 31-35, 2010.

6. Zhang X, Siegelman ES, Lee MK, Todon R. Pancreatic schwannoma, an extremely rare and challenging entity: report of two cases and review of literature. Pancreatology 19: 729-737, 2019.

7. Morita S, Okuda J, Taketani M, et al. Pancreatic schwannoma: report of a case. Surg Today 29: 1093-1097, 1999.

8. Wu YL, Yan HC, Chen LR, et al. Pancreatic benign schwannoma treated by simple enucleation: case report and review of literature. Pancreas 31: 286-288, 2005.

9. Yu RS, Sun JZ. Pancreatic schwannoma: CT findings. Abdom Imaging 31: 103-105, 2006.

10. Li S, Ai SZ, Owens C, Kulesza P. Intrapancreatic schwannoma diagnosed by endoscopic ultrasound-guided fine-needle aspiration cytology. Diagn Cytopathol 37: 132-135, 2009.

11. Dorsey F, Taggart MW, Fisher WE. Image of the month. Pancreatic schwannoma. Arch Surg 145: 913-914, 2010.

12. Devi J, Sathyalakshmi R, Chandramouleeswari K, Devi NR. Pancreatic schwannoma-a rare case report. J Clin Diagn Res 8: 15-16, 2014.

13. Antonini F, Santinelli A, Macarri G. Endoscopic ultrasound-guided fine-needle aspiration of an unusual pancreatic mass. Clin Gastroenterol Hepatol 13: e25, 2015.

14. Nishikawa T, Shimura K, Tsuyuguchi T, Kiyono S, Yokosuka O. Contrast-enhanced harmonic EUS of pancreatic schwannoma. Gastrointest Endosc 83: 463-464, 2016.

15. Xu SY, Sun K, Gyabaah K, et al. Central pancreatectomy for pancreatic schwannoma: a case report and literature review. World $\mathrm{J}$ Gastroenterol 22: 8439-8446, 2016.

16. Sung S, Rao R, Sharaiha RZ. Fine-needle aspiration cytology of pancreatic schwannoma. Diagn Cytopathol 45: 668-670, 2017.

17. Fukuhara $S$, Fukuda $S$, Tazawa $H$, et al. A case report of pancreatic schwannoma showing increased FDG uptake on PET/CT. Int J Surg Case Rep 36: 161-166, 2017.

18. Doxtader EE, Sturgis CD, Dyhdalo KS. Cystic pancreatic schwannoma diagnosed by endoscopic ultrasound-guided fine needle aspiration. Diagn Cytopathol 46: 883-885, 2018.

19. Wang H, Zhang BB, Wang SF, et al. Pancreatic schwannoma: imaging features and pathological findings. Hepatobiliary Pancreat Dis Int 19: 200-202, 2020.

20. Javed AA, Wright MJ, Hasanain A, et al. Pancreatic nerve sheath tumors: a single institutional series and systematic review of the literature. J Gastrointest Surg 24: 841-848, 2020.

21. Bruno M, Maletta F, Gaia S, et al. Wait-and-see policy for a small pancreatic schwannoma diagnosed with endoscopic ultrasound with fine-needle aspiration. ACG Case Rep J 6: 1-3, 2019.

22. Wang S, Xing C, Wu H, Dai M, Zhao Y. Pancreatic schwannoma mimicking pancreatic cystadenoma: a case report and literature review of the imaging features. Medicine (Baltimore) 98: e16095, 2019.

23. Azami T, Takano Y, Niiya F, et al. A case of pancreatic schwannoma diagnosed by endoscopic ultrasound-fine needle aspiration. Clin J Gastroenterol 13: 585-590, 2020.

24. Mukai S, Itoi $\mathrm{T}$, Yamaguchi $\mathrm{H}$, et al. A retrospective histological comparison of EUS-guided fine-needle biopsy using a novel Franseen needle and a conventional end-cut type needle. Endosc Ultrasound 8: 50-57, 2019.

25. Okuwaki K, Masutani H, Kida M, et al. Diagnostic efficacy of white core cutoff lengths obtained by EUS-guided fine-needle biopsy using a novel 22G Franseen biopsy needle and sample isolation processing by stereomicroscopy for subepithelial lesions. Endosc Ultrasound 9: 187-192, 2020. 
26. Moriya T, Kimura W, Hirai I, et al. Pancreatic schwannoma: case report and an updated 30-year review of the literature yielding 47 cases. World J Gastroenterol 18: 1538-1544, 2012.
The Internal Medicine is an Open Access journal distributed under the Creative Commons Attribution-NonCommercial-NoDerivatives 4.0 International License. To view the details of this license, please visit (https://creativecommons.org/licenses/ by-nc-nd/4.0/).

(C) 2021 The Japanese Society of Internal Medicine Intern Med 60: 1389-1395, 2021 